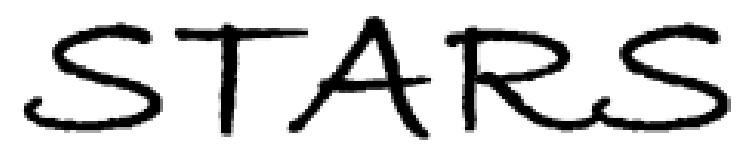

University of Central Florida

STARS

$1-1-2014$

\title{
Analysis of adiabatic trapping for quasi-integrable area-preserving maps
}

Armando Bazzani

Christopher Frye

University of Central Florida

Massimo Giovannozzi

Cédric Hernalsteens

Find similar works at: https://stars.library.ucf.edu/facultybib2010

University of Central Florida Libraries http://library.ucf.edu

This Article is brought to you for free and open access by the Faculty Bibliography at STARS. It has been accepted for inclusion in Faculty Bibliography 2010 s by an authorized administrator of STARS. For more information, please contact STARS@ucf.edu.

\section{Recommended Citation}

Bazzani, Armando; Frye, Christopher; Giovannozzi, Massimo; and Hernalsteens, Cédric, "Analysis of adiabatic trapping for quasi-integrable area-preserving maps" (2014). Faculty Bibliography 2010s. 5045. https://stars.library.ucf.edu/facultybib2010/5045

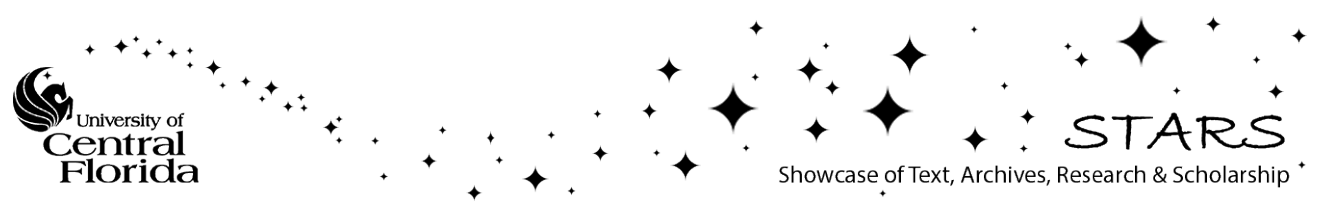




\title{
Analysis of adiabatic trapping for quasi-integrable area-preserving maps
}

\author{
Armando Bazzani* \\ Physics and Astronomy Department, Bologna University, V. Irnerio 46, Bologna, Italy \\ Christopher Frye $\mathrm{e}^{\dagger}$ \\ Department of Physics and Department of Mathematics, University of Central Florida, Orlando, Florida, USA \\ and Beams Department, CERN, 1211 Geneva 23, Switzerland \\ Massimo Giovannozzi $i^{\ddagger}$ \\ Beams Department, CERN, 1211 Geneva 23, Switzerland \\ Cédric Hernalsteens ${ }^{\S}$ \\ Beams Department, CERN, 1211 Geneva 23, Switzerland and EPFL, LPAP, 1015 Lausanne, Switzerland
}

(Received 6 November 2013; revised manuscript received 26 January 2014; published 25 April 2014)

\begin{abstract}
Trapping phenomena involving nonlinear resonances have been considered in the past in the framework of adiabatic theory. Several results are known for continuous-time dynamical systems generated by Hamiltonian flows in which the combined effect of nonlinear resonances and slow time variation of some system parameters is considered. The focus of this paper is on discrete-time dynamical systems generated by two-dimensional symplectic maps. The possibility of extending the results of neo-adiabatic theory to quasi-integrable areapreserving maps is discussed. Scaling laws are derived, which describe the adiabatic transport as a function of the system parameters using a probabilistic point of view. These laws can be particularly relevant for physical applications. The outcome of extensive numerical simulations showing the excellent agreement with the analytical estimates and scaling laws is presented and discussed in detail.
\end{abstract}

DOI: 10.1103/PhysRevE.89.042915

PACS number(s): 05.45.-a, 45.10.Hj, 45.20.Jj

\section{INTRODUCTION}

The neo-adiabatic theory [1-12] has been developed to estimate the change of the adiabatic invariant when separatrixcrossing phenomena occur in slowly modulated one-degreeof-freedom Hamiltonian systems. The theory provides explicit formulas for the trapping probabilities in a resonance region [2], for the change of the adiabatic invariant due to separatrix crossing, and for the error estimate defining the regions of validity in phase space [3].

Adiabatic transport is also possible under these conditions. An ensemble of particles trapped into a resonance region can be moved at a distance of order $O(1)$ at a time $O(1 / \epsilon)$, where $\epsilon$ is the adiabatic parameter that defines the slow time scale. The adiabatic transport by means of nonlinear resonances has relevant applications in plasma physics $[8,13]$, accelerator physics [14-17], and celestial mechanics [18-20] and, in general, for controlling a particle distribution under the effect of nonlinear dynamics. It is also worth mentioning that even if plasma and accelerator physics are the typical domains of applicability of adiabatic theory, fields as diverse as quantum systems, nanostructures, and superconductors are also dealing with problems of adiabatic transport (see, e.g., Refs. [21-23]).

The application of the theoretical results to physical experiments encounters the problem of a quantitative evaluation of the theory's limits and of the extension of the analytical results

\footnotetext{
*armando.bazzani@bo.infn.it

${ }^{\dagger}$ christopher.frye@knights.ucf.edu

${ }^{\ddagger}$ massimo.giovannozzi@cern.ch

$\S$ cedric.hernalsteens@cern.ch
}

to realistic models. In several situations the theory suggests the existence of simple relations among physical observables, which can be extended to very generic situations due to their robust character. Along this line, we perform analytical and numerical studies to derive scaling laws for the efficiency of the adiabatic trapping and transport in quasi-integrable Hamiltonian systems. In particular we consider the possibility to extend the neo-adiabatic theory to analytic area-preserving maps in the neighborhood of a stable elliptic fixed point, for which, to the best of our knowledge, no rigorous result exists yet.

The numerical simulations show that such scaling laws are robust and they apply to a wide class of models even if a rigorous extension of the neo-adiabatic invariance theory is not possible due to the presence of infinite nonlinear resonances in phase space $[24,25]$.

This is the case for quasi-integrable systems like the Hénon map [26], which is a relevant model to study nonlinear effects in celestial mechanics and accelerator physics [27]. To cope with the problem of nonintegrability we take advantage of the existence of an interpolating Hamiltonian, i.e., a Hamiltonian whose phase flux interpolates at an integer times the orbits of the maps in the neighborhood of the elliptic fixed point with an error that can be exponentially small in the distance from the elliptic fixed point [28].

The interpolating Hamiltonian can be perturbatively computed using Birkhoff normal forms [27,29,30]. To extend, at least partially, the neo-adiabatic theory to area-preserving maps we have to consider the effect of the discrete time dependence, which implies the existence of an infinite number of resonances in phase space. In this paper it is shown that the formulas for continuous time can also be applied to discrete-time systems, as long as the definition of the improved adiabatic 
invariant (IAI) is modified to control the dynamics near the separatrix curve. This requires a cutoff in the Fourier expansion in the action-angle variables to be introduced. Moreover, thanks to the theoretical considerations outlined here, which set a rigorous framework for the analysis of discrete-time systems, it is possible to compute explicitly the dependence of the trapping efficiency from the adiabatic parameter and the properties of the resonance under consideration.

It is worth mentioning that numerical simulations on slowly modulated Hamiltonian systems have been performed by various authors, mainly to study the weak chaotic regions swept by a moving resonance and the diffusive behavior of orbits in phase space [9,31-33]. In this paper, however, we adopt a different and original point of view. On one hand, we use numerical simulations to study the limits of the theoretical results, which require specific conditions on the adiabatic parameters and the resonance structure. Also, we aim at evaluating the efficiency of adiabatic transport under different situations relevant for applications. On the other hand, the numerical simulations allow us to define specific protocols of time variation of the free parameters of the system under consideration, and this is a crucial point in view of optimizing the adiabatic transport in the presence of specific requirements, e.g., the control of the final particle distribution in phase space such as in Refs. [34-36]. It is worth stressing that the points addressed in this paper are extremely relevant in applications, particularly in the field of beam physics, where crossing a nonlinear resonance has been proposed as a means to split the beam in the transverse phase space [14-17] to perform a multiturn extraction from a circular particle accelerator. This novel technique requires accurate control of the intensity sharing between the various beamlets as well as of the losses during transport of the trapped beamlets. Therefore, theoretical and robust scaling laws describing the detail of the trapping process are essential for optimizing the actual beam manipulation.

The plan of this paper is as follows: in Sec. II some results of the neo-adiabatic theory for Hamiltonian flows are presented and extended to discrete-time systems (maps) as required for our study. The analysis of pendulum-like systems is presented in Sec. III, discussing the details of the trapping process, its optimization, and the efficiency of transport of the trapped initial conditions. Analytical area-preserving maps, a generalization of the Hénon map, are dealt with in Sec. IV, where the details of the trapping process are studied, with particular emphasis on the dependence of the fraction of trapped orbits on the distribution of initial conditions and on the system's parameters. Furthermore, scaling laws for the size of the phase space area where trapping into resonance cannot occur are also presented and discussed in detail. Finally, some conclusions are drawn in Sec. V, and in the Appendix a number of results used in the main text are collected.

\section{ADIABATIC THEORY AND TRAPPING INTO RESONANCE}

\section{A. Adiabatic theory for quasi-integrable area-preserving maps}

The extension of adiabatic theory to area-preserving maps has to address a number of specific issues due to the discontinuous nature of their time dependence. Indeed, if one considers a slowly modulated area-preserving map written in the form

$$
\left(q_{n+1}, p_{n+1}\right)=\mathcal{M}\left(q_{n}, p_{n}, \epsilon n\right), \quad \epsilon \ll 1,
$$

a discontinuous change in the dynamics occurs at each iteration. Letting $\lambda=\epsilon n$, we initially assume that the frozen map is integrable, so that there exists a Hamiltonian $H(q, p, \lambda)$ such that

$$
\mathcal{M}(q, p, \lambda)=\exp \left[D_{H(q, p, \lambda)}\right](q, p),
$$

where the operator $D_{H(q, p, \lambda)}$ is the Lie derivative defined using the Poisson bracket $[\cdot, \cdot]$ as

$$
\begin{aligned}
D_{H(q, p, \lambda)} f(q, p) & =[f(q, p), H(q, p, \lambda)] \\
& =\sum_{i} \frac{\partial f}{\partial q_{i}} \frac{\partial H}{\partial p_{i}}-\frac{\partial f}{\partial p_{i}} \frac{\partial H}{\partial q_{i}} .
\end{aligned}
$$

As a consequence, $H(q, p, \epsilon t)$ is an interpolating Hamiltonian for map (1) with an error of order $O(\epsilon)$; that is, the phase flow associated with $H(q, p, \epsilon t)$ interpolates the orbits $\left(q_{n}, p_{n}\right)$ up to an error $O(\epsilon)$. This fact prevents the possibility of applying directly the results of adiabatic theory for Hamiltonian systems to the modulated map (1). In the Appendix we show how the existence of an interpolating Hamiltonian allows an extension of the adiabatic theory to maps of the type in (1) under suitable conditions. By using the generating function $F(q, I, \lambda)$ [see Eq. (A1) in the Appendix] to compute the action-angle variables $(\theta, I)$ for the frozen system, it is possible to write the modulated map (2) in the form

$$
\begin{aligned}
\mathcal{M}\left(\theta_{n}, I_{n}, \lambda\right)= & \exp \left[\epsilon D_{\partial F / \partial \lambda}\left(\theta_{n}, I_{n}, \lambda\right)\right] \exp \left[D_{H\left(I_{n}, \lambda\right)}\right]\left(\theta_{n}, I_{n}\right) \\
& +O\left(\epsilon^{2}\right), \quad \lambda=\epsilon n .
\end{aligned}
$$

Then perturbation theory allows us to introduce improved action-angle variables $(\phi, J)$, such that the modulated map (3) reads

$$
\begin{aligned}
\phi_{n+1} & =\phi_{n}+\Omega\left(J_{n}, \phi_{n}, n \epsilon\right)+O\left(\epsilon^{2}\right), \\
J_{n+1} & =J_{n}+O\left(\epsilon^{2}\right),
\end{aligned}
$$

as long as no resonances

$$
k \Omega(I, \lambda)=2 \pi h, \quad k, h \in \mathbb{Z}
$$

are present in the phase space region under consideration, also taking into account the values spanned by varying $\lambda$, provided $|k| \leqslant k_{\max }$, where $k_{\max }$ is an appropriate cutoff (see the Appendix). To control the evolution of the adiabatic invariant up to the separatrix we have to show that the cutoff error does not depend on the distance to the separatrix curve. This is indeed the case, and the details of the approach to be used to prove these statements is given in the Appendix.

In Sec. IV we show, using numerical simulations, that the previous results can be extended to area-preserving maps in the neighborhood of an elliptic fixed point. Indeed, in this case the Birkhoff normal forms theory suggests the existence of an interpolating Hamiltonian for the frozen map in the neighborhood of the elliptic fixed point, whose error becomes exponentially small $\propto \exp \left[-\left(r_{0} / r\right)^{\eta}\right]$ with respect to the distance $r$ from the elliptic fixed point when $r \rightarrow 0$ 
[28], where $r_{0}, \eta$ are suitable positive constants depending on the arithmetic properties of the linear frequency. Even if the explicit calculation of the optimal interpolating Hamiltonian is not possible, the perturbative approach based on Birkhoff normal forms allows us to point out the dependence of the phase space structure on the map parameters. Therefore, according to the previous assumptions, by considering the neighborhood of the origin where the error is $O\left(\epsilon^{3}\right)$, we can prove the existence of an IAI for the modulated map.

\section{B. Trapping into resonance and change of adiabatic invariant when crossing a separatrix}

According to Birkhoff normal forms, the interpolating Hamiltonian for an analytic area-preserving map in the neighborhood of an elliptic fixed point can be written in the form [27]

$$
H(\rho, \psi, \lambda)=H_{0}(\rho, \lambda)+A(\lambda) \rho^{m / 2} \cos m \psi+O\left(\rho^{m+1}\right),
$$

where $m$ is the order of the resonance under consideration and

$$
\rho=\frac{q^{2}+p^{2}}{2}, \quad \psi=\operatorname{atan} \frac{q}{p} .
$$

Without loss of generality we assume that

$$
H_{0}(\rho, \lambda) \simeq \omega_{1}(\lambda) \rho+\frac{\omega_{2}(\lambda)}{2} \rho^{\hat{m}},
$$

where $\omega_{2}(\lambda)<0$ and $\omega_{1}(\lambda)$ is a monotonic function, satisfying $\omega_{1} \in[-\mu, \mu]$ when $\lambda \in[0,1]$. The applicability of adiabatic theory to this time-dependent, quasi-integrable map is discussed at the end of the Appendix. If $\hat{m}<m$, then the resonance is stable; otherwise, it is unstable, as the separatrices can pass through the origin. Most of the computations reported in this paper refer to $m=4$ and $\hat{m}=2$.

The existence of real, positive solutions in $\rho$ to the equation $\partial H_{0} / \partial \rho=0$ for fixed $\lambda$ implies the existence of separatrix curves for the frozen system, and we distinguish three different regions in phase space (see also Fig. 1, left): (i) the region above the resonance islands (region I), (ii) the region below the resonance islands (region II), and (iii) the region inside the resonance islands (region III). According to the remarks in the previous section and in the Appendix, we can compute an IAI for an adiabatically modulated map in the form [see Eq. (A16)

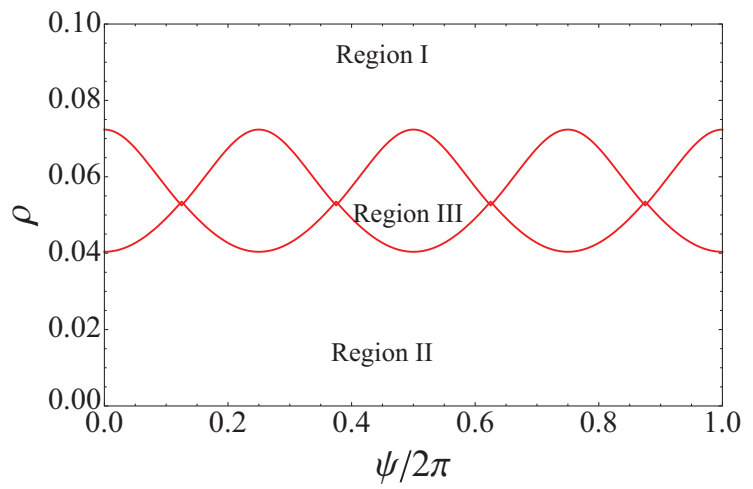

for the definition of the operator $\mathbb{T}_{\Omega}$ ]

$$
J=I+\frac{\epsilon}{2 \pi} \int_{0}^{\mathcal{T}}\left(\frac{\mathcal{T}}{2}-t\right) \mathbb{T}_{\Omega}\left\{\frac{\partial H}{\partial \lambda}\right\}_{\leqslant k_{\max }(\Omega(I, \lambda), \epsilon)} d t
$$

where $\mathcal{T}(I, \lambda)$ is the orbit period of the frozen system and $\{\cdot\}_{k}$ stands for the truncation of the Fourier expansion to order $k$, using the same approach as for Hamiltonian systems. The change of the adiabatic invariant due to separatrix crossing is estimated applying Neishtadt's theory [3-5] since the IAI (9) tends to the IAI of the interpolating Hamiltonian. Moreover, the evolution of the frozen energy $H(\rho, \psi, \lambda)=E$ and of the scaled period $\epsilon \mathcal{T}(J, \lambda)$ can be described by the dynamics of the interpolating Hamiltonian up to an error $O\left(\epsilon^{2}\right)$.

We are interested in describing the time evolution of an ensemble of particles initially distributed in region I of the phase space under the effect of surface increase, induced by the change of parameter $\lambda$, of both regions II and III. Following Neishtadt, we consider the phase space areas $\Sigma_{\text {II,III }}(\lambda)$ enclosed by the separatrix curves in regions II and III, which are both bounded, and we define

$$
\Sigma_{\mathrm{I}}=\Sigma_{\mathrm{II}}+\Sigma_{\mathrm{III}}, \quad \frac{d \Sigma_{i}}{d \lambda}=\Theta_{i}(\lambda)>0 \quad i=\mathrm{I}, \mathrm{II}, \mathrm{III} ;
$$

then $\Theta_{\mathrm{I}}=\Theta_{\mathrm{II}}+\Theta_{\mathrm{III}}$ also represents the derivative of the surface of region I, but with opposite sign. The condition $\Theta_{\text {II,III }}>0$ is mandatory to have a nonzero trapping probability in regions II and III, as they are growing during the resonance crossing process.

For each particle, we introduce the so-called crossing parameter $\lambda_{*}$ according to the equation $\Sigma_{\mathrm{I}}\left(\lambda_{*}\right)=2 \pi J_{-}$, where $J_{-}$is the initial value of the invariant $J$ in region I. The existence of the crossing parameter implies that the particle can enter into either region II or III by the effect of the separatrix crossing.

When the adiabatic theory holds, it is possible to prove that the transition probability $P$ from region I to regions II and III is given by

$$
P_{\mathrm{I} \rightarrow \mathrm{II}}=\frac{\Theta_{\mathrm{II}}}{\Theta_{\mathrm{I}}}, \quad P_{\mathrm{I} \rightarrow \mathrm{III}}=\frac{\Theta_{\mathrm{III}}}{\Theta_{\mathrm{I}}} .
$$

The transition phenomenon induced by the separatrix crossing is described in a probabilistic way $[4,5,37]$ by using the random

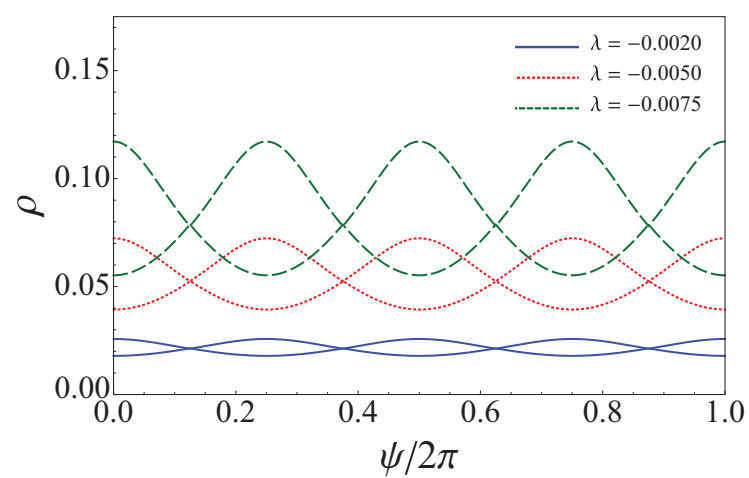

FIG. 1. (Color online) Schematic view of the regions generated by the separatrix of the frozen system (6) (left) and the position of the separatrix for the same system, but including the $\lambda$ dependence (right). 
variables

$$
\xi_{\mathrm{I}}=\frac{\left|h_{0}^{\mathrm{I}}\right|}{\epsilon \Theta_{\mathrm{I}}}, \quad \xi_{\mathrm{II}}=\frac{\left|h_{0}^{\mathrm{II}}\right|}{\epsilon \Theta_{\mathrm{II}}},
$$

where $h_{0}^{\mathrm{I}}$ and $h_{0}^{\mathrm{II}}$ are the orbit energy computed via the interpolating Hamiltonian at Poincaré sections of phase space just before entering region III and just after entering region II, respectively. It turns out that the variables $\xi_{i}$ are uniformly distributed in the interval $[0,1]$, and they are quite sensitive to the value of $J_{-}$. Moreover, the theory is correctly applied only when $K \sqrt{\epsilon}<\xi_{i}<1-K \sqrt{\epsilon}$ for a suitable positive constant $K$. This condition allows an easy estimate of the fraction of particles whose evolution is not described by the adiabatic theory. Once the separatrix crossing phenomenon occurs, one can compute the change of the IAI in the new phase space region. For the transition $\mathrm{I} \rightarrow \mathrm{III}$ in a generic case we have the estimate

$$
\begin{aligned}
2 \pi J_{+}-\Sigma_{\mathrm{III}}\left(\lambda_{*}\right)= & -\epsilon \alpha \Theta_{\mathrm{III}}\left(\xi_{\mathrm{I}}-\frac{1}{2}\right) \\
& \times\left(\ln \epsilon \Theta_{\mathrm{I}}-\frac{2 \Theta_{\mathrm{I}}}{\Theta_{\mathrm{III}}} \ln \epsilon \Theta_{\mathrm{III}}\right)+O(\epsilon),
\end{aligned}
$$

where $J_{+}$is the final value of the IAI and $\alpha$ is the inverse of the logarithm of the eigenvalue of the frozen map at the unstable hyperbolic fixed point. Similarly, the transition I $\rightarrow$ II gives a change in the IAI of

$$
\begin{aligned}
2 \pi J_{+}-\Sigma_{\mathrm{II}}\left(\lambda_{*}\right)= & -\epsilon \alpha \Theta_{\mathrm{II}}\left(\xi_{\mathrm{II}}-1\right) \\
& \times\left(\ln \epsilon \Theta_{\mathrm{I}}+\frac{\Theta_{\mathrm{I}}}{\Theta_{\mathrm{II}}} \ln \epsilon \Theta_{\mathrm{II}}\right)+O(\epsilon) .
\end{aligned}
$$

In the previous estimates we have only reported the leading terms of order $O(\epsilon \ln \epsilon)$ in a generic system (for a more detailed result see Refs. [3,31]) since our goal is to describe the adiabatic trapping in the resonance region for an ensemble of particles. Referring to the phase space structure of Hamiltonian (6), we assume that when the parameter $\lambda$ is varied, the resonance region is enlarged and moved outwards (see Fig. 1, right). As a consequence the areas of regions II and III increase, and an orbit starting in region I can be trapped in region II or III provided that adiabatic theory applies. An orbit starting in region I tends to preserve the IAI value during the slow variation of $\lambda$ until it reaches the separatrix when $\lambda=\lambda_{*}$. Then, it is possible to describe the separatrix crossing phenomenon if the orbit is not too close to the hyperbolic point (condition on the $\xi_{i}$ variables) neglecting terms of order $O\left(\epsilon^{3 / 2}\right)$ and the IAI performs a pseudostochastic dynamics according to Eqs. (13) and (14).

Some conditions need to be fulfilled for the adiabatic theory to be applicable. For Hamiltonian (6) we define the adiabatic parameter $\varepsilon$ as the ratio between $\epsilon$ and the square of the secondary frequency $\omega_{\mathrm{e}}^{2}$, i.e., the frequency of small oscillations around the elliptic fixed point inside the resonance region. When the adiabatic parameter $\varepsilon$ is $O(1)$, one cannot justify estimates (13) and (14). As a consequence, we lose the control of the adiabatic invariant at the separatrix crossing, and the adiabatic trapping into resonances is not possible. When the resonance is stable and $\omega_{1} \ll \omega_{2}$ [see Eq. (8)], a perturbative approach [27] applied to Hamiltonian (6) provides the estimates for the frequency of the elliptic fixed points

$$
\omega_{\mathrm{e}}^{2} \simeq \frac{A}{\omega_{2}}\left(\frac{\omega_{1}}{\omega_{2}}\right)^{m / 2}
$$

whereas the resonance distance from the origin is

$$
\rho_{\mathrm{e}} \propto \sqrt{\frac{\omega_{1}}{\omega_{2}}} .
$$

Then the smallness condition on the adiabatic parameter reads

$$
\varepsilon=\frac{\epsilon}{\omega_{\mathrm{e}}^{2}} \propto \frac{\epsilon \omega_{2}}{\rho_{\mathrm{e}}^{m}} \ll 1 \Rightarrow \epsilon \ll \frac{\rho_{\mathrm{e}}^{m}}{\omega_{2}},
$$

and we derive the following scaling law for the minimum distance of the resonance from the origin, which allows the trapping phenomenon to start (trapping radius in the following):

$$
R_{\min } \propto \epsilon^{1 / m} .
$$

In a similar way one can prove that if condition (15) holds, then the change of the IAI is small compared to the area of resonance region III, which also scales as $\rho_{\mathrm{e}}^{m / 2}(\lambda)$ for Hamiltonian (6).

The situation changes for the case of unstable resonances. As an example, we consider the third-order resonance $(m=3)$, for which the interpolating Hamiltonian is approximated by

$$
H \simeq-\omega_{1} \rho-A \rho^{3 / 2} \cos 3 \psi+\omega_{2} \frac{\rho^{2}}{2}
$$

and the hyperbolic fixed points are located at $\psi_{\mathrm{h}}=\pi / 3$ and at a distance

$$
\rho_{\mathrm{h}}=-\frac{3 A}{4 \omega_{2}}+\sqrt{\left(\frac{3 A}{4 \omega_{2}}\right)^{2}+\frac{\omega_{1}}{\omega_{2}}} \simeq \frac{2 \omega_{1}}{3 A},
$$

so that $\rho_{\mathrm{h}} \propto \omega_{1}$, which is the frequency of the elliptic fixed point at the origin. The condition on the adiabatic parameter reads

$$
\frac{\epsilon}{\omega_{1}^{2}} \ll 1
$$

and the radius $\rho_{\mathrm{h}}$ satisfies the scaling law

$$
\frac{\epsilon}{\rho_{\mathrm{h}}^{2}} \ll 1 \Rightarrow R_{\min } \propto \epsilon^{1 / 2} .
$$

For a generic unstable resonance of order $m$ we can perform similar calculations, obtaining a scaling law for the minimum radius

$$
R_{\min } \propto \epsilon^{1 / 2(m-2)} .
$$

The trapping efficiency can be evaluated considering that the theory applies to the orbits not passing too close to the hyperbolic fixed points, i.e., $\xi_{i} \in[K \sqrt{\varepsilon}, 1-K \sqrt{\varepsilon}]$. Therefore, whenever $\Theta_{\text {III }}>0$, the trapping efficiency is given by

$$
c_{\mathrm{I} \rightarrow \mathrm{III}}(\lambda)=\frac{\Theta_{\mathrm{III}}(\lambda)}{\Theta_{\mathrm{I}}(\lambda)}(1-2 K \sqrt{\varepsilon}) .
$$


Letting $n(\rho)$ be the radial density of the ensemble of particles, the total number of trapped particles will be given by

$$
N_{\mathrm{III}}=\int_{\lambda_{0}}^{\lambda_{1}} n(\rho(\lambda)) \frac{\Theta_{\mathrm{III}}(\lambda)}{\Theta_{\mathrm{I}}(\lambda)}\left[1-2 K \frac{\sqrt{\epsilon}}{\omega_{\mathrm{e}}(\lambda)}\right] d \lambda,
$$

and we have the relation

$$
N_{\text {III }}=c_{0}-c_{1} \sqrt{\epsilon}=c_{0}-c_{1} \sqrt{\frac{\left|\lambda_{1}-\lambda_{0}\right|}{T}},
$$

where $T=\left|\lambda_{1}-\lambda_{0}\right| / \epsilon$ is the time interval over which the trapping process takes place. It is worth stressing that, indeed, the lower limit of integration $\lambda_{0}$ might need to be replaced by $\min \left(\lambda_{0}, R_{\min }\right)$ to take into account the loss of adiabaticity close to the origin of the phase space. This phenomenon occurs also for the particles that enter into region II from region I. In fact, a fraction proportional to

$$
c_{\mathrm{I} \rightarrow \mathrm{II}}(\lambda)=\frac{\Theta_{\mathrm{II}}(\lambda)}{\Theta_{\mathrm{I}}(\lambda)}(1-2 K \sqrt{\varepsilon})
$$

changes the IAI according to the theory, whereas the other particles may be scattered in phase space [19].

The particles not trapped may feature a large variation in the adiabatic invariant, thus changing the particles' distribution in phase space. This point is essential for our considerations. In fact, if the initial distribution is strongly affected by the change of IAI during the crossing process, then the estimate provided by Eq. (20) (and similarly for $N_{\text {II }}$ ) is no longer correct, as $n(\rho)$ should also account for the dynamical change of shape during the resonance crossing process.

\section{ADIABATIC TRANSPORT FOR PENDULUM-LIKE SYSTEMS}

In order to study the parametric dependence of the adiabatic transport in this section we consider pendulum-like Hamiltonian systems whose Hamiltonian function has the form

$$
H(\theta, I, \lambda)=\frac{1}{2}[I-\delta(\lambda)]^{2}-[1+\beta(\lambda)] \cos \theta,
$$

where $\delta, \beta$ are functions with $\beta(\lambda)>-1$. This Hamiltonian has also been considered in Ref. [38] to study transport due to resonance trapping. The expression for the fixed points is given by

$$
I=\delta(\lambda), \quad \theta=n \pi \quad n \in \mathbb{Z},
$$

and it is easy to find that for $n=0$ the fixed point is elliptic, while for $n=1$ it is hyperbolic. The equation of the separatrix emanating from the hyperbolic fixed point reads

$$
I_{ \pm}^{*}(\lambda, \theta)=\delta(\lambda) \pm 2 \cos \left(\frac{\theta}{2}\right) \sqrt{1+\beta(\lambda)},
$$

while the area of the stable island and its $\lambda$ derivative is given by

$$
\Sigma_{\mathrm{III}}=16 \sqrt{1+\beta(\lambda)} \quad \Theta_{\mathrm{III}}=\frac{8 \dot{\beta}(\lambda)}{\sqrt{1+\beta(\lambda)}} .
$$

The last quantity that is relevant for our analysis is the angular frequency of oscillation around the elliptic fixed point, which is equal to

$$
\omega_{\mathrm{e}}(\lambda)=\sqrt{1+\beta(\lambda)} .
$$

The meaning of the auxiliary functions $\delta$ and $\beta$ is clear: $\delta(\lambda)$ represents the shift along the $I$ axis of the fixed point, while $\beta(\lambda)$ is related to the size of the stable island. Therefore, these two parameters allow us to control the resonance position and size in an independent way. This is an essential feature of this model, which enables an optimal control of the global dynamics to allow an accurate assessment of the impact of the island growth and transport on the trapping phenomenon. Unfortunately, such an independent control is lost in the case of the area-preserving maps that will be considered in Sec. IV.

\section{A. Analysis of trapping efficiency}

To illustrate the analytic results and their predictive power we consider a rather complex variation of the free parameters of the pendulum-like system in order to mimic what could be an optimized trapping and transport process. A uniform initial distribution of particles given by

$$
n(\theta, I)=\left\{\begin{array}{cc}
N / 2 \pi & \text { for }(\theta, I) \in[-\pi, \pi] \times[0,1], \\
0 & \text { otherwise }
\end{array}\right.
$$

has been used in the numerical simulations. We will also let $\delta$ increase linearly from $\delta(0)=0.5$ to $\delta(1)=1.5$ during a time $T=1 / \epsilon$, where $\lambda=\epsilon t=t / T$, and

$$
\delta(\lambda)=\frac{1}{2}+\lambda .
$$

Furthermore, $\beta$ will increase quadratically from $\beta(0)=\beta_{i}$ (if $\beta_{i}=-1$, the stable island begins as a slit with zero size at the center of the initial conditions) to some $\beta(1)=\beta_{f}$ which we keep arbitrary for now:

$$
\beta(\lambda)=\left(\beta_{f}-\beta_{i}\right) \lambda^{2}+\beta_{i} .
$$

A key quantity that will be considered throughout this paper is the so-called trapping fraction $\tau$, which is defined as the ratio between the initial conditions that are trapped into the nonlinear resonance and the total number of initial conditions. For the case under consideration, after some algebra and assuming a uniform distribution of initial conditions and a perfect adiabaticity of the process, which corresponds to neglecting the correction factor depending on $K$ in $c_{\mathrm{I} \rightarrow \mathrm{III}}, c_{\mathrm{I} \rightarrow \mathrm{II}}$ and computing the integral in Eq. (20) while carefully retaining the sign of $\Theta_{i}$ in the case of shrinking regions, then the estimate of $\tau$ reads

$$
\tau=\left\{\begin{array}{cc}
\frac{4 \sqrt{1+\beta_{f}}}{\pi+4 \sqrt{1+\beta_{f}}} & \text { for }-1 \leqslant \beta_{f} \leqslant \frac{\pi^{2}}{16}-1, \\
\frac{4}{\pi} \sqrt{1+\beta_{f}}-\frac{1}{2} & \text { for } \frac{\pi^{2}}{16}-1 \leqslant \beta_{f} \leqslant \frac{9 \pi^{2}}{64}-1, \\
1 & \text { for } \beta_{f} \geqslant \frac{9 \pi^{2}}{64}-1 .
\end{array}\right.
$$

This prediction is depicted as a solid line in Fig. 2.

We set up simulations with parameters identical to those described above, while setting $T$ to be 30,100 , and 3500 turns. The results of the numerical simulations are shown in Fig. 2 as a series of symbols of different colors for the different values of $T$. The agreement between the numerical simulations and the prediction improves as a function of $T=1 / \epsilon$. For shorter $T$ the motion of the separatrix is not adiabatic, and the trapping is shown to be less efficient in the part where $\tau$ varies quadratically. However, when $\tau$ varies linearly, the trapping is 


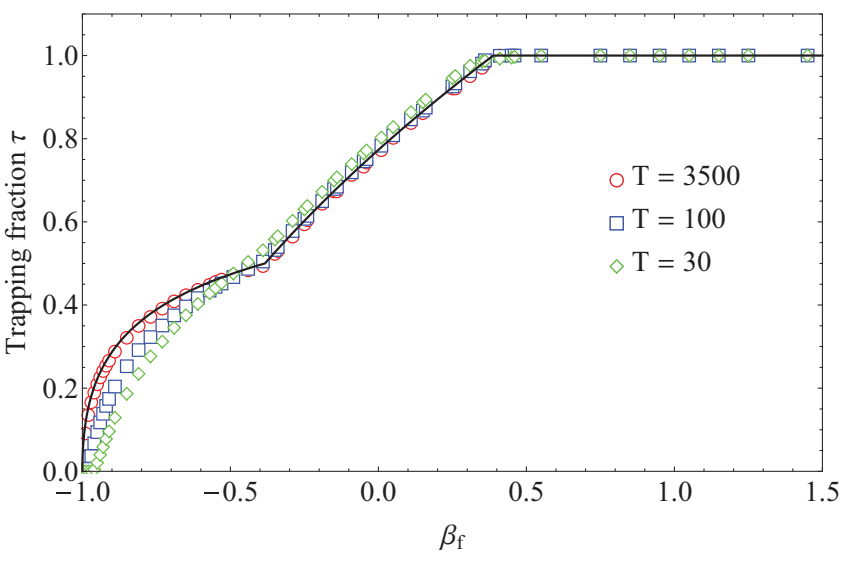

FIG. 2. (Color online) The results of the trapping fraction study when we let $\delta(\lambda)$ increase linearly while $\beta(\lambda)$ increases quadratically. The curve represents the predicted trapping fraction, while the symbols stand for the results of numerical simulations. A close match between predictions and simulation results is visible for $T=3500$.

even higher than the theoretical prediction. This might be due to the impact of the nonadiabaticity of the initial part of the trapping process, which could have generated higher-density regions in the distribution, e.g., in the tails. This, in turn, could lead to an apparent increase of trapping efficiency since in the theoretical model the particle distribution is assumed to be constant throughout the whole process.

\section{B. Optimization of the trapping process}

With the previous case we showed that the theory is capable of describing the adiabatic trapping phenomenon with generic variation of the free parameters of Hamiltonian (23). This opens up the possibility of performing an optimization of the overall trapping process.

The first step consists of exploiting the possibility of independently controlling the island position and its surface. The ideal case could be a fixed, but growing, island, which would trivially trap all initial conditions intercepted by the expanding separatrix.

Another possibility consists of fixing the value of $\Theta_{i}$ in order to impose a well-defined trapping probability. Once more, the presence of two free parameters can be used for this purpose. It is easy to see that the phase space area beneath the stable island varies in time as

$$
\Theta_{\mathrm{II}}=2 \pi \dot{\delta}-\frac{\Theta_{\mathrm{III}}}{2} .
$$

Therefore, if $\Theta_{\mathrm{II}}$ is set to zero, then $c_{\mathrm{I} \rightarrow \mathrm{II}}$ will be zero too, thus providing a full trapping into region III. The condition to impose is

$$
\dot{\delta}=\frac{\Theta_{\mathrm{III}}}{4 \pi}=\frac{2 \dot{\beta}(\lambda)}{\pi \sqrt{1+\beta(\lambda)}} .
$$

Integrating with respect to $\lambda$, we find

$$
\delta(\lambda)=\delta_{0}+\frac{4}{\pi} \sqrt{1+\beta(\lambda)},
$$

where we have assumed that $\beta(0)=-1$, corresponding to zero initial size for the island. This relation can also be inverted to give $\beta(\lambda)$ with the required dependence on $\delta(\lambda)$ :

$$
\beta(\lambda)=\frac{\pi^{2}}{16}\left[\delta(\lambda)-\delta_{0}\right]^{2}-1 .
$$

It is worth noting that since the area of region II remains constant, particles in that region must remain there since passing the separatrix would result in a decrease in the adiabatic invariant orbit area. Therefore, under these conditions the trapping process will satisfy the following relations:

$$
N_{\mathrm{II}}=N_{\mathrm{II}}^{0}, \quad N_{\mathrm{III}}=N_{\mathrm{III}}^{0}+N_{\mathrm{I}}^{0} .
$$

These considerations have been probed by a number of numerical simulations, whose results are presented in Fig. 3. Figure 3(a) refers to simulations performed with $10^{5}$ initial conditions distributed uniformly over $[-\pi, \pi] \times[2.2,2.4]$ and where both $\delta(\lambda), \beta(\lambda)$ vary linearly in time in such a way that both $\Theta_{\text {II }}, \Theta_{\text {III }}>0$. The results are qualitatively as expected with particles in both regions II and III.

The remaining three plots refer to simulations with $\delta(\lambda)$ and $\beta(\lambda)$ varying according to relationship (35). Depending on where the island is created, it is possible to share equally the initial conditions in regions II and III [Fig. 3(d)] or to have particles only in region III [Fig. 3(b)]. Finally, in Fig. 3(c) a case in which $\beta(\lambda)$ is varying more slowly than imposed by Eq. (35) is shown, which should simulate a nonoptimal control of the system parameters. Of course, in this case some initial conditions are trapped in region II.

\section{Transport efficiency}

We are also interested in testing how efficiently a moving resonance can hold onto the particles undergoing libration around its point of stable equilibrium. This aspect is interesting as it could be combined with the trapping phenomena to transport towards higher values of $I$ the conditions initially trapped into the islands. To this aim we define the transport efficiency $\nu(\epsilon)$ as

$$
\nu(\epsilon)=\frac{N_{\mathrm{III}}^{f}}{N_{\mathrm{III}}^{i}},
$$

where $N_{\mathrm{III}}^{i}$ and $N_{\mathrm{III}}^{f}$ stand for the number of particles trapped in region III at the beginning and at the end of the resonance transport, respectively. The theory predicts (see below) that a simple power law should exist between the transport efficiency $\nu(\epsilon)$ and the adiabatic parameter $\epsilon / \omega_{\mathrm{e}}^{2}$.

To test this, we set up initial conditions for $10^{5}$ particles in the rectangle $[-\pi, \pi] \times[0,1]$ and let the stable island begin as a zero-size slit at $I=1 / 2$. Then, we let the island grow adiabatically until achieving a given area and thus capturing a given number of particles proportional to this area. Subsequently, we let the island move at various speeds by changing $\delta$ from $1 / 2$ to 3 .

The results for many different final island sizes $\Sigma_{\mathrm{III}} \in$ $[0,2 \pi]$ and various moving speeds, represented by the adiabatic parameter in the plot, are displayed in Fig. 4. A fit through the region between $10 \%$ and $60 \%$ of transport efficiency, in order to probe the regime where adiabatic theory applies, 

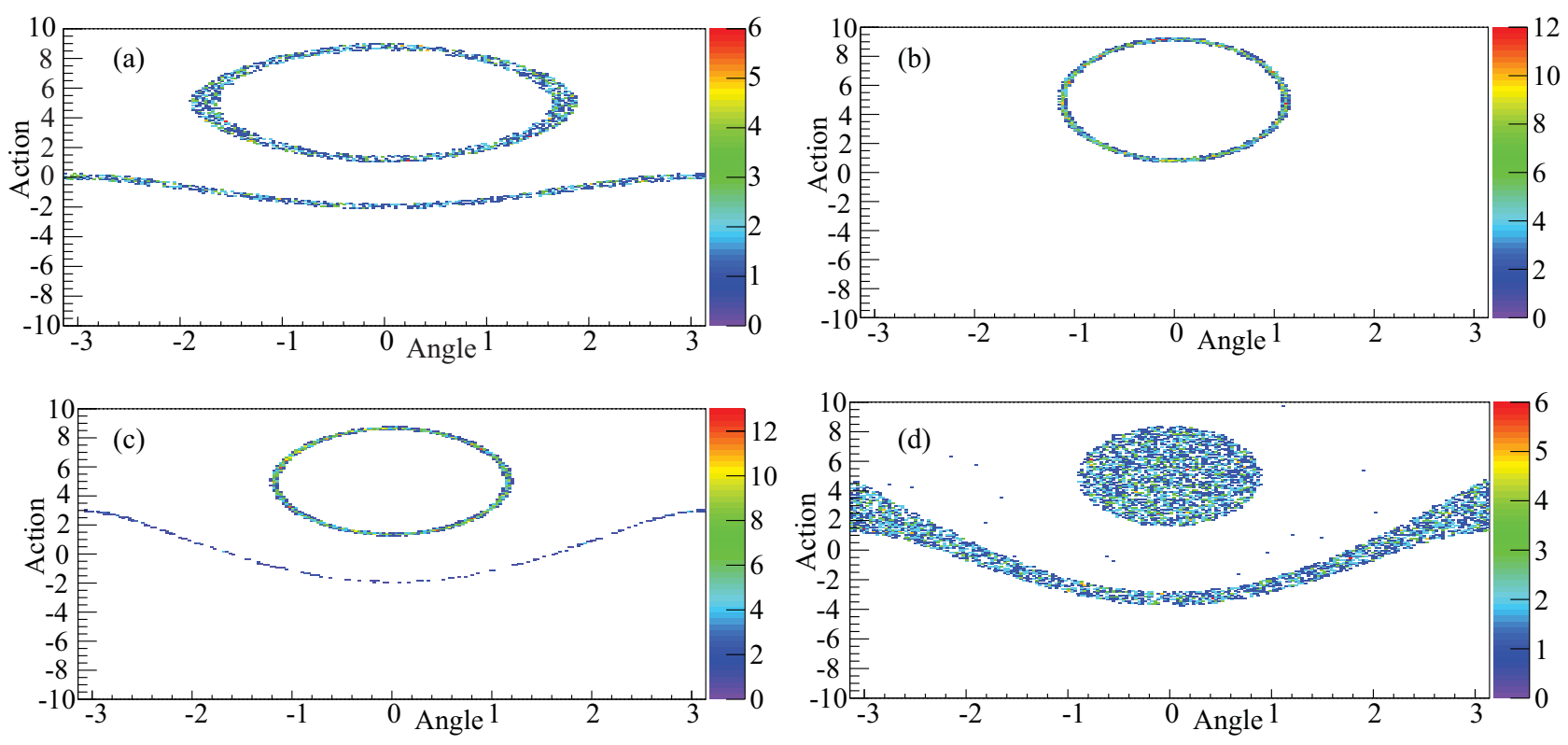

FIG. 3. (Color online) Examples of final distributions generated from the same initial distribution and different relationships between $\beta$ and $\delta$ during the trapping process. (a) Transport of a growing island, obtained by a linear variation of both $\delta(\lambda)$ and $\beta(\lambda)$. (b) $100 \%$ trapping into resonance obtained by using Eq. (35), $\delta(\lambda)=5 \lambda / 3000$, and a resonance starting just below the initial distribution. (c) Less than $100 \%$ trapping into resonance obtained by using the same parameters as (b), but with $\beta(\lambda)$ increasing more slowly, and a resonance starting just below the initial distribution. (d) $50 \%$ trapping into resonance obtained by using the same parameters as (b) and a resonance starting in the middle of the initial distribution.

provides a simple power law:

$$
1-v(\epsilon)=(1.132 \pm 0.004)\left(\frac{\epsilon}{\omega_{\mathrm{e}}^{2}}\right)^{0.754 \pm 0.003} .
$$

It is interesting to investigate how detrapping from the island region, also indicated as loss of particles, is distributed during the transport part of the process. The simulation results are shown in Fig. 5, where the losses as a function of turn number are depicted for several values of the adiabatic parameter. It is clearly seen that they occur at the very beginning of such a

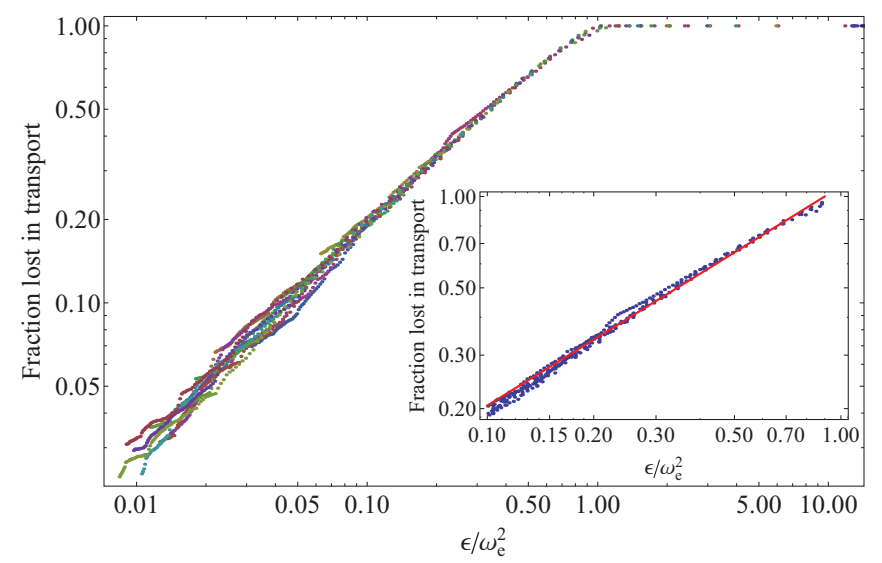

FIG. 4. (Color online) Transport efficiency results after an island, full of particles in libration, moves at various speeds. The various curves refer to different values of the final island's size $\Sigma_{\text {III. The }}$ numerical results can be interpolated by a power-law dependence $\propto \epsilon^{3 / 4}$ of the transport efficiency as a function of the adiabatic parameter as shown in the inset. stage; hence, only the trapped particles close to the separatrix are lost during transport, and no mechanism of refilling this region is acting during the whole transport process.

The pendulum-like Hamiltonian (23) allows us to infer a simple interpretation for this scaling law. Since the parameter $\delta(\lambda)$ is varied linearly during transport, whereas the parameter $\beta$ is kept constant, one can perform the canonical change of variables associated with the generating function $G(J, \theta, \lambda)=$ $[J+\delta(\lambda)] \theta$, and the new Hamiltonian turns out to be that of a forced pendulum, where the forcing term is proportional to the adiabatic parameter $\epsilon$,

$$
H(\theta, J, \lambda)=\frac{1}{2} J^{2}-[1+\beta] \cos \theta+\dot{\delta}(\lambda) \theta .
$$

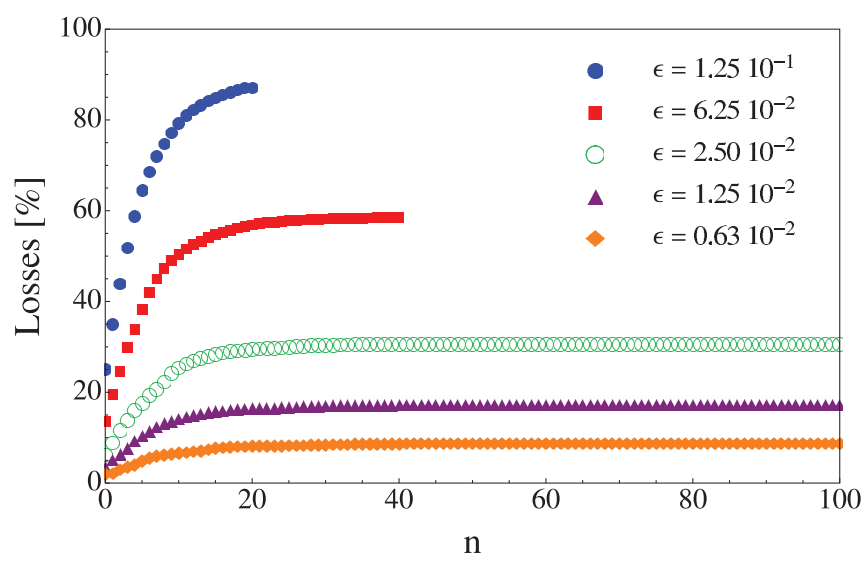

FIG. 5. (Color online) Time evolution of the losses during the transport stage of the process under study. 
In the new variables, the phase space area $\mathcal{A}$ of the stability region around the elliptic fixed point is reduced by terms of order $O(\epsilon|\ln \epsilon|)$, and we expect that all particles contained in this area can be transported, whereas those outside are quickly lost. Neglecting the contribution of the logarithm, this result suggests a direct proportionality between the fraction of lost particles and the adiabatic parameter. Therefore, the empirical scaling law $v(\epsilon) \propto \epsilon^{3 / 4}$ for the transport efficiency (see Fig. 4) could be a consequence of a nonuniform distribution of trapped particles due to the change of trapping efficiency during the modulation of the $\beta$ parameter and to the slow dynamics close to the separatrix. When the adiabatic parameter tends to 1 , the stability region of Hamiltonian (38) shrinks to the origin, so that all particles are lost and no transport is possible. Despite the simplicity of the pendulum-like systems, similar mechanisms are observed in generic models, such as the Hénon map.

In terms of control of the adiabatic trapping and transport, this result suggests that the strategy of separating the process into two very distinct phases might not be the best option. In fact, the possible advantage of generating a growing but standing island in terms of trapping efficiency might be lost due to the losses appearing during the separate transport stage.

\section{ADIABATIC TRAPPING FOR AREA-PRESERVING MAPS}

The second class of models under consideration is a generalization of the quadratic polynomial 2D map, the socalled Hénon map [26]. The map reads

$$
\left(\begin{array}{l}
q \\
p
\end{array}\right)_{n+1}=R(\omega)\left(\begin{array}{c}
q \\
p+q^{2}+\kappa q^{3}
\end{array}\right)_{n}
$$

where $R(\omega)$ is a two-dimensional (2D) rotation matrix of an angle $\omega$ and $\kappa \in \mathbb{R}$.

The corresponding interpolating Hamiltonian [27] is of the form (6), which, tailored to the case of the fourth-order resonance $(m=4)$, reads

$$
H(\psi, \rho, \lambda)=\lambda \rho+\frac{\omega_{2}(\lambda)}{2} \rho^{2}+\lambda\left|u_{0,3}(\lambda)\right| \rho^{2} \cos 4 \psi,
$$

in which

$$
\omega_{2}(\lambda)=-\frac{1}{16}\left[3 \cot \frac{\omega(\lambda)}{2}+\cot \frac{3 \omega(\lambda)}{2}+6 \kappa\right]
$$

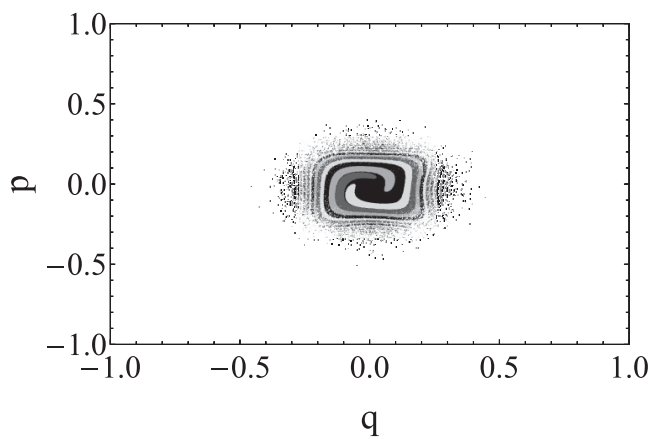

and

$$
u_{0,3}(\lambda)=\frac{1}{16}\left[\cot \frac{\omega(\lambda)}{2}-\cot \frac{3 \omega(\lambda)}{2}-2 \kappa\right],
$$

with

$$
\omega(\lambda)=\lambda+\frac{\pi}{2}, \quad \lambda=\frac{\Delta \omega}{T} t .
$$

The fixed points satisfy the following conditions:

$$
\rho_{+}(\lambda)=-\frac{\lambda}{\omega_{2}(\lambda)+2\left|u_{0,3}(\lambda)\right| \lambda}, \quad \psi_{+}=k \frac{\pi}{2}
$$

or

$$
\rho_{-}(\lambda)=-\frac{\lambda}{\omega_{2}(\lambda)-2\left|u_{0,3}(\lambda)\right| \lambda}, \quad \psi_{-}=\frac{\pi}{4}+k \frac{\pi}{2} .
$$

Since the coordinate $\rho$ is non-negative, we see that for the fixed points to exist we need the condition $\lambda \omega_{2}(\lambda)<0$. Furthermore, the stability analysis shows that the fixed points $\left(\psi_{+}, \rho_{+}\right)$are stable, while $\left(\psi_{-}, \rho_{-}\right)$are unstable.

The separatrices have the form

$$
\rho_{\text {sep }}^{ \pm}(\lambda, \psi)=\frac{-\lambda \pm 2 \sqrt{\frac{\left|u_{0,3}(\lambda)\right| \lambda^{3} \cos ^{2} 2 \psi}{2\left|u_{0,3}(\lambda)\right| \lambda-\omega_{2}(\lambda)}}}{\omega_{2}(\lambda)+2\left|u_{0,3}(\lambda)\right| \lambda \cos 4 \psi},
$$

and the surface of one island out of the chain of four is given by

$$
\begin{aligned}
\Sigma_{\mathrm{III}}= & 2 \sqrt{\frac{\lambda^{2}}{4 \lambda^{2}\left|u_{0,3}(\lambda)\right|^{2}-\omega_{2}^{2}(\lambda)}} \\
& \times \tanh ^{-1} \sqrt{\frac{4 \lambda\left|u_{0,3}(\lambda)\right|}{\omega_{2}(\lambda)+2 \lambda\left|u_{0,3}(\lambda)\right|}} .
\end{aligned}
$$

Finally, the angular frequency of oscillations around the elliptic fixed points is equal to

$$
\omega_{\mathrm{e}}(\lambda)=4 \lambda \sqrt{\left|\frac{\lambda u_{0,3}(\lambda)}{\omega_{2}(\lambda)}\right|} .
$$

This model is more complex than the pendulum-like model. First of all, some symmetries are lost, as is the case for the separatrices, for which the lower and upper separatrix branches are no longer symmetric. Furthermore, the fact that all the coefficients of Hamiltonian (6) are $\lambda$ dependent implies that

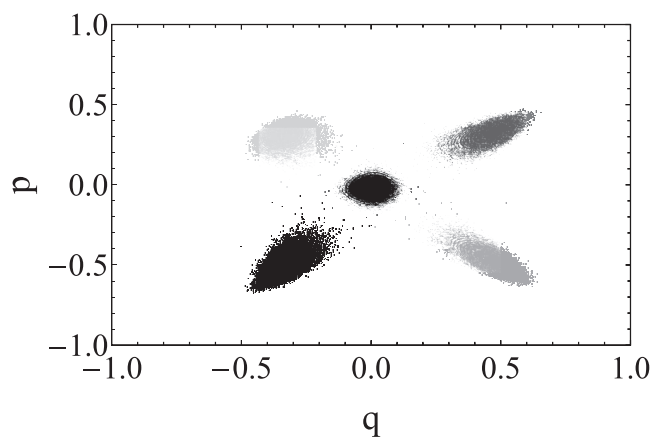

FIG. 6. Details of the trapping phenomenon for map (39) with $\kappa=-1.1, \Delta \omega / T \approx 4.4 \times 10^{-6}$, and a Gaussian initial distribution of $5 \times 10^{5}$ particles with $\sigma=0.1$. The initial conditions (left) have been color-coded in order to identify in which island they will eventually be trapped (right). 


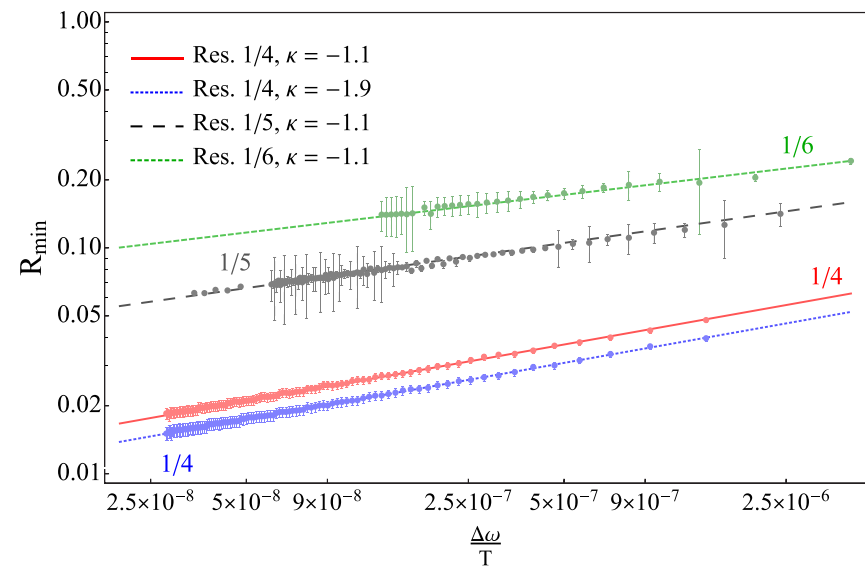

FIG. 7. (Color online) $R_{\min }$ as a function of the adiabatic parameter $\Delta \omega / T$ in log-log scale. Different stable resonances and values of the parameter $\kappa$ are shown together with the fit functions in which the exponents have been fixed according to the theory. The agreement with the proposed scaling law $a(\Delta \omega / T)^{1 / m}$ is remarkable.

this parameter simultaneously affects the position and size of the island. A possibility to overcome this difficulty would be to use the additional free parameter $\kappa$ as an additional tuning knob, making it a function of $\lambda$. This option has not been considered yet.

The main features of the trapping process can be seen in Fig. 6. The initial conditions have been identified on the basis of the location at the end of the trapping process. It is clearly seen that the islands start trapping only at a finite distance from the origin. Then, in a given amplitude interval a well-defined area in phase space is trapped in the islands. At even larger amplitudes a chaotic region appears, and initial conditions arbitrarily close can end up in different islands.

The numerical studies presented in the following section aim at probing the quantitative aspects of the scaling law of the no-trapping area around the origin and of the trapping efficiency.

\section{A. Analysis of $\boldsymbol{R}_{\text {min }}$}

It is clear that $R_{\min }$ is essential for any application aiming at a well-defined sharing of particles between the islands and core. Simulations have been performed using a uniform distribution of initial conditions and determining the fraction of trapped particles as a function of the radius $R$ of the initial

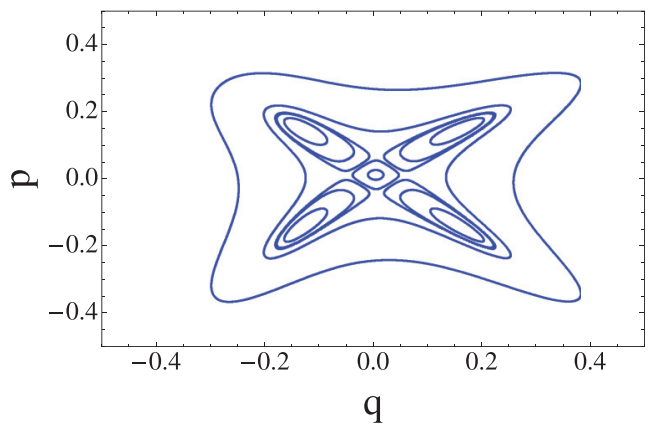

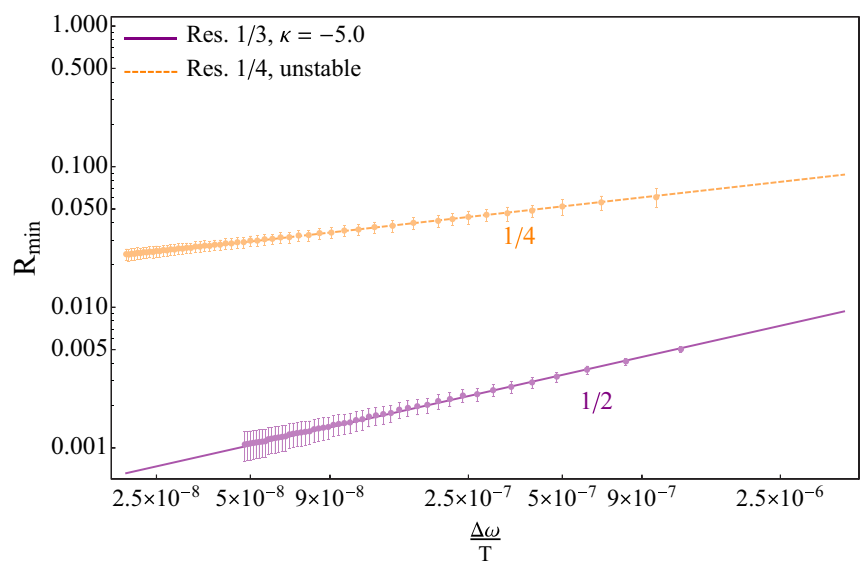

FIG. 9. (Color online) $R_{\min }$ as a function of the adiabatic parameter $\Delta \omega / T$ in log-log scale. The $1 / 3$ and $1 / 4$ unstable resonances have been used. The curves represent the fit curves $a(\Delta \omega / T)^{b}$. The exponents of the theoretical scaling law are also reported. The agreement between scaling law and numerical simulations is remarkable.

distribution. A fine scan over $R$ has been performed, together with a fit of the computed trapping fraction to estimate its zero crossing, which corresponds to $R_{\min }$. This procedure has been repeated for several values of $T$, and the resulting function $R_{\min }(T)$ is shown in Fig. 7 together with fit functions based on the scaling (16) for several values of the parameter $\kappa$ and also resonance order $m$. The log-log plot shows excellent agreement between the scaling law and the numerical results.

Additional numerical tests have been performed using the $1 / 3$ and $1 / 4$ unstable resonances. Indeed, while the first is generically unstable [27], the latter can be either stable or unstable. In our case, a modified version of map (39) has been used, where the parameters controlling the strength of the nonlinear terms have been used to set $\omega_{2}(\lambda)=0$ in Hamiltonian (6), which corresponds to turning the origin unstable, as described in Ref. [39]. In this case the control parameter $\lambda$ has been changed in order to shrink the separatrix down to the origin, which is possible due to the unstable character of the resonance. Hence, the trapping process is somewhat different from the one considered so far. An example of the phase space topology for the $m=4$ stable and unstable resonances is shown in Fig. 8. The results of the numerical simulations for the computation of $R_{\min }(T)$ are shown in Fig. 9.

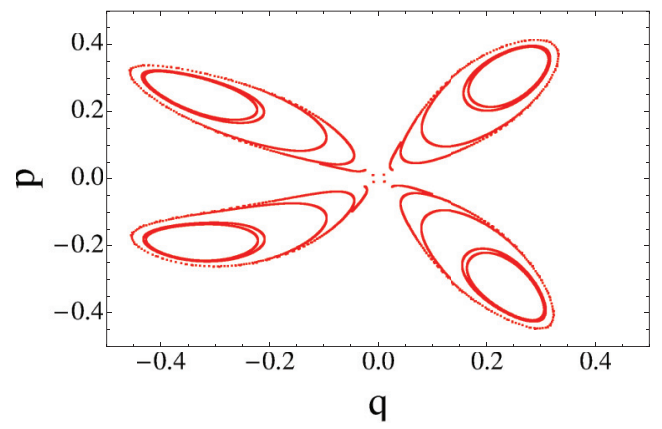

FIG. 8. (Color online) Example of phase space portraits for map (39) close to the stable resonance with $m=4$ (left) and its unstable version (right) based on the approach described in Ref. [39]. 
TABLE I. Summary of the fit parameters of the scaling law $a / T^{b}$ for the evolution of $R_{\min }$.

\begin{tabular}{lcccc}
\hline \hline Stability type & Resonance order & $\kappa$ & $a \pm \Delta a$ & $b \pm \Delta b$ \\
\hline Stable & $1 / 4$ & -1.1 & $1.31 \pm 0.08$ & $0.246 \pm 0.003$ \\
Stable & $1 / 4$ & -1.9 & $1.11 \pm 0.08$ & $0.247 \pm 0.005$ \\
Stable & $1 / 5$ & -1.1 & $1.51 \pm 0.09$ & $0.184 \pm 0.003$ \\
Stable & $1 / 6$ & -1.1 & $1.6 \pm 0.3$ & $0.15 \pm 0.01$ \\
Unstable & $1 / 3$ & -5.0 & $3 \pm 1$ & $0.48 \pm 0.01$ \\
Unstable & $1 / 4$ & N.A. & $1.7 \pm 0.4$ & $0.24 \pm 0.01$ \\
\hline \hline
\end{tabular}

This scaling law agrees with the theoretical prediction given by Eq. (18).

The summary of the fit parameters for both stable and unstable resonances is given in Table I for the fit functions $a / T^{b}$. In the case of the stable $1 / 4$ resonance it has been possible to derive a scaling law for the fit parameter $a$, which is $a(\kappa)=a_{0}+a_{1} \kappa$, with $a_{0}=0.3 \pm 0.1, a_{1}=1.6 \pm$ 0.2 obtained by analyzing numerical simulations for $-1.9 \leqslant$ $\kappa \leqslant-1.1$ in steps of 0.1 .

\section{B. Analysis of trapping efficiency}

An extensive campaign of numerical simulations has been performed, with the parameter $\kappa$ scanned, as well as $T$. As far as the initial distribution is concerned, both Gaussian and uniform functions have been used, performing scans over their rms widths.

The results of numerical simulations are reported in Fig. 10 (top), where the trapping fraction as a function of $T$ is plotted for several values of $\sigma$ of the Gaussian distribution and for two values of $\kappa$. The numerical data have been fitted using the scaling law (21) and have been added to the plot as solid lines. The agreement is remarkable, and some discrepancy is visible only for the case referring to the smallest value of $\sigma$. It is important to point out that from the considerations of the previous section, where $R_{\min }(T)$ has been discussed, the lower limit of the integral in (20) has to be modified to take into account that no trapping can occur for amplitudes smaller than $R_{\min }$. Moreover, as $R_{\min }$ depends on the adiabatic parameter, the trapping process will be affected differently as a function of $T$. Furthermore, as $\sigma$ becomes smaller, the impact of $R_{\min }(T)$ becomes larger; this explains why the agreement between the numerical data and the scaling law gets worse for small values of $\sigma$. However, such an effect can be exactly quantified. The first step consists of computing the value $\lambda_{\min }$ corresponding to $R_{\min }$, which is given by

$$
2 \pi R_{\min }^{2}=\int_{0}^{2 \pi} \rho_{\text {sep }}^{+}\left(\lambda_{\min }, \psi\right) d \psi .
$$

As $R_{\min } \ll 1$, it is possible to develop Eq. (49) and retain only the lower-order term in $\lambda_{\text {min }}$, thus obtaining

$$
\lambda_{\min } \propto R_{\min }^{2} \propto \epsilon^{2 / m},
$$

where the last step is valid in the case of stable resonances. The expression for $N_{\text {III }}$ can be reanalyzed by considering that in our simulations the lower limit of integration can be assumed to be zero, but the effect of $R_{\min }$ has to be taken into account.
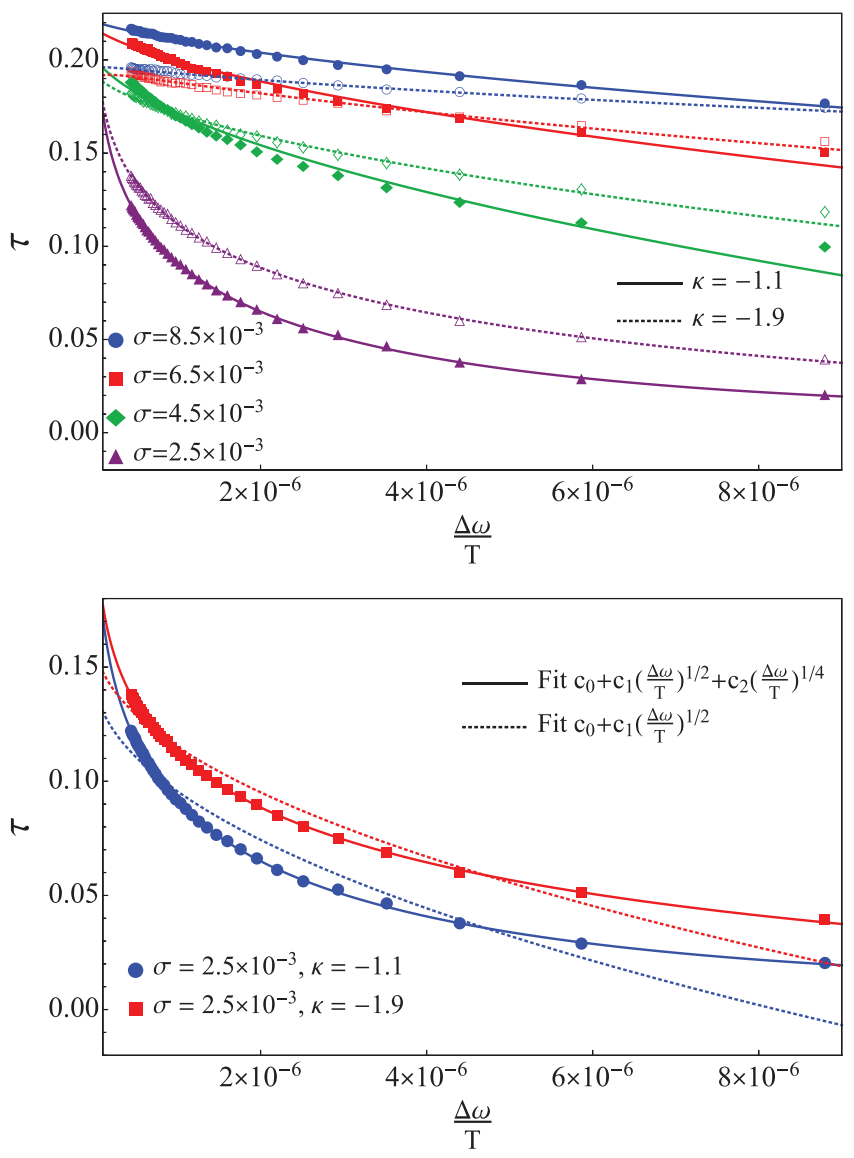

FIG. 10. (Color online) (top) Trapping fraction $\tau$ [see Eq. (31)] as a function of the adiabatic parameter $\Delta \omega / T$ for different values of $\kappa$ and $\sigma$ of Gaussian distributions. The solid symbols and solid lines refer to the case $\kappa=-1.1$, while the open symbols and the dotted lines refer to the case $\kappa=-1.9$. The fit curves are in very good agreement with the numerical data. (bottom) Comparison of fit curves with (solid lines) and without (dotted lines) the effect of $R_{\min }(T)$ for the simulations performed with smaller $\sigma$. The agreement is remarkable.

Hence, Eq. (20) can be recast in the form

$$
\begin{aligned}
N_{\mathrm{III}}= & \int_{\lambda_{\min }}^{\lambda_{1}} n(\rho(\lambda)) \frac{\Theta_{\mathrm{III}}(\lambda)}{\Theta_{\mathrm{I}}(\lambda)}\left[1-2 K \frac{\sqrt{\epsilon}}{\omega_{\mathrm{e}}(\lambda)}\right] d \lambda \\
= & \int_{0}^{\lambda_{1}} n(\rho(\lambda)) \frac{\Theta_{\mathrm{III}}(\lambda)}{\Theta_{\mathrm{I}}(\lambda)}\left[1-2 K \frac{\sqrt{\epsilon}}{\omega_{\mathrm{e}}(\lambda)}\right] d \lambda \\
& -\int_{0}^{\lambda_{\min }} n(\rho(\lambda)) \frac{\Theta_{\mathrm{III}}(\lambda)}{\Theta_{\mathrm{I}}(\lambda)}\left[1-2 K \frac{\sqrt{\epsilon}}{\omega_{\mathrm{e}}(\lambda)}\right] d \lambda \\
\approx & c_{0}-c_{1} \epsilon^{1 / 2}+c_{2} \lambda_{\min }+c_{3} \lambda_{\min }^{-1 / 2} \epsilon^{1 / 2} \\
\approx & c_{0}-c_{1} \epsilon^{1 / 2}+c_{2} \epsilon^{1 / 2}+c_{3} \epsilon^{1 / 2-1 / m} .
\end{aligned}
$$

The term $c_{3} \lambda_{\min }^{-1 / 2} \epsilon^{1 / 2}$ is generated by the scaling $\omega_{\mathrm{e}}(\lambda) \approx$ $\lambda^{-3 / 2}$, which can be derived from Eq. (48), while the last step of Eq. (51) is based on the estimate (50). In the particular case $m=4$ the scaling law for $N_{\text {III }}$ simplifies to $N_{\text {III }} \approx c_{0}+c_{1} \epsilon^{1 / 2}+c_{2} \epsilon^{1 / 4}$, with the redefinition of the symbols $-c_{1}+c_{2} \rightarrow c_{1}$ and $c_{3} \rightarrow c_{2}$. This prediction has been tested using the data referring to numerical simulations 
with the smaller $\sigma$ shown in Fig. 10 (top). The results are shown in Fig. 10 (bottom). For the sake of comparison, the fit function obtained by neglecting the effect of $R_{\min }$ is also shown as dotted lines. The improvement in the agreement between numerical data and theoretical prediction is clearly visible.

\section{CONCLUSIONS}

In this paper we have briefly reviewed the theory of adiabatic trapping and transport for Hamiltonian systems and presented an extension suitable for applications to discretetime systems, i.e., area-preserving modulated maps. We have explicitly considered two different classes of systems, namely, a pendulum-like Hamiltonian and a Hénon map, to compare the analytical results with numerical simulations. The first class allows studying the parametric dependence of the trapping phenomenon, whereas by means of the second class we face the problem of extending the theoretical predictions to quasi-integrable discrete-time systems.

Our main goal is to understand the dependence of adiabatic trapping and transport efficiency on the system parameters and to propose robust scaling laws suitable for extending to more general models, relevant for physical applications.

Given the broad range of domains in which adiabatic trapping and transport play a crucial role, these results might be particularly relevant for applications. In particular, the results of these studies can be used in the process of optimizing adiabatic transport or of mitigating the effects of unavoidable resonance crossing by proper control of the crossing process. It is worth stressing that these topics are of paramount importance, e.g., in the domain of particle accelerators, where novel beam manipulations have been proposed based on adiabatic transport.

By means of extensive numerical simulations the scaling laws ruling the adiabatic transport and trapping have been verified, and the agreement between predictions and numerical results is excellent. These laws allow us to understand and explore the domain of validity of the theory, which is essential to shed light on the details of the trapping mechanism.

Even if these results have been obtained for rather generic systems, in terms of the form of the underlying Hamiltonian, the dimensionality of the phase space is still too low and hence represents a limit to the applicability of our findings to realistic physical models. Therefore, the next step will be to attempt to extend these results to Hamiltonian systems with two degrees of freedom, where a richer phase space topology might lead to new phenomena.

\section{ACKNOWLEDGMENTS}

We would like to express our warm gratitude to A. Neishtadt for discussions and valuable comments on the topic dealt with in this paper. We would also like to thank J. Williams for useful comments on the original manuscript. We are particularly thankful to the referees for the excellent comments that allowed us to improve the original manuscript. One of the authors (A.B.) would like to thank the Accelerator and
Beam Physics Group of CERN Beams Department for the hospitality.

\section{APPENDIX: ADIABATIC INVARIANCE FOR MODULATED AREA-PRESERVING MAPS}

To extend the adiabatic theory to the modulated map (3) we apply perturbation theory by introducing action-angle variables in each region defined by the separatrix curves. Let us define the generating function

$$
F(q, E, \lambda)=\int_{H(q, p, \lambda)=E}^{q} p(\hat{q}, E, \lambda) d \hat{q},
$$

where $E=H(I, \lambda)$ is expressed as a function of the action variable

$$
I(E, \lambda)=\frac{1}{2 \pi} \oint_{H(q, p, \lambda)=E} p(q, E, \lambda) d q .
$$

We perform the change of variables in the modulated map $\mathcal{M}$ (1) according to

$$
\mathcal{N}(\epsilon n)=T^{-1}(\epsilon(n+1)) \circ \mathcal{M}(\epsilon n) \circ T(\epsilon n),
$$

where the symbol $\circ$ indicates the composition of functions and the transformation $T(\epsilon n):\left(\theta_{n}, I_{n}\right) \rightarrow(q, p)$ is implicitly defined by

$$
\begin{aligned}
p & =\left.\frac{\partial F}{\partial q}\right|_{I}\left(q, I_{n}, \epsilon n\right), \\
\theta_{n} & =\left.\frac{\partial F}{\partial I}\right|_{q}\left(q, I_{n}, \epsilon n\right) .
\end{aligned}
$$

Note that the variables $(p, q)$ are uniquely defined, whereas the definition of the variables $\left(\theta_{n}, I_{n}\right)$ depends explicitly on $n$ since the Hamiltonian function changes. According to our assumptions, Eq. (A3) can be written in the form

$$
\mathcal{N}(\epsilon n)=T^{-1}(\epsilon(n+1)) \circ T(\epsilon n) \circ \exp \left[D_{H(I, \epsilon n)}\right],
$$

and we explicitly compute the map $T^{-1}(\epsilon(n+1)) \circ T(\epsilon n)$ tangent to the identity from the relations

$$
\begin{aligned}
p= & \frac{\partial F}{\partial q}\left(q, I_{n+1}, \epsilon(n+1)\right)=\frac{\partial F}{\partial q}\left(q, I_{n}, \epsilon n\right) \\
& +\left.\epsilon \frac{\partial}{\partial \lambda}\right|_{q, p} \frac{\partial F}{\partial q}\left(q, I_{n}, \epsilon n\right)+O\left(\epsilon^{2}\right), \\
\theta_{n+1}= & \frac{\partial F}{\partial I}\left(q, I_{n+1}, \epsilon(n+1)\right)=\frac{\partial F}{\partial I}\left(q, I_{n}, \epsilon n\right) \\
& +\left.\epsilon \frac{\partial}{\partial \lambda}\right|_{q, p} \frac{\partial F}{\partial I}\left(q, I_{n}, \epsilon n\right)+O\left(\epsilon^{2}\right),
\end{aligned}
$$

where $I_{n}=I(q, p, \epsilon n)$ and, when not explicitly written, the partial derivatives are computed using $F=F(q, I, \lambda)$. From 
definition (A4), algebraic calculations give

$$
\begin{aligned}
& \frac{\partial^{2} F}{\partial q \partial \lambda}+\frac{\partial^{2} F}{\partial q \partial I} \frac{\partial I}{\partial \lambda}=0, \\
& \frac{\partial^{2} F}{\partial I^{2}}+\frac{\partial^{2} F}{\partial I \partial q} \frac{\partial q}{\partial I}=0,
\end{aligned}
$$

and from these relations we obtain

$$
\begin{aligned}
\left.\frac{\partial}{\partial \lambda}\right|_{q, p} \frac{\partial F}{\partial I} & =\frac{\partial^{2} F}{\partial I \partial \lambda}+\frac{\partial^{2} F}{\partial I^{2}} \frac{\partial I}{\partial \lambda} \\
& =\frac{\partial^{2} F}{\partial I \partial \lambda}+\frac{\partial^{2} F}{\partial q \partial \lambda} \frac{\partial q}{\partial I}=\left.\frac{\partial}{\partial I}\right|_{\theta} \frac{\partial F}{\partial \lambda} .
\end{aligned}
$$

The second equation of the system (A4) reads

$$
\theta^{\prime}=\theta+\left.\epsilon \frac{\partial}{\partial I}\right|_{\theta} \frac{\partial F}{\partial \lambda}+O\left(\epsilon^{2}\right)
$$

where we identify $\theta^{\prime}=\theta_{n+1}$ and $I=I_{n}$. Finally, the map $T^{-1}(\epsilon(n+1)) \circ T(\epsilon n)$ can be obtained by imposing the symplecticity conditions

$$
\begin{aligned}
& \theta^{\prime}=\theta+\left.\epsilon \frac{\partial}{\partial I}\right|_{\theta} \frac{\partial F}{\partial \lambda}+O\left(\epsilon^{2}\right), \\
& I^{\prime}=I-\left.\epsilon \frac{\partial}{\partial \theta}\right|_{I} \frac{\partial F}{\partial \lambda}+O\left(\epsilon^{2}\right),
\end{aligned}
$$

and it is related to the phase flow at time $\epsilon$ of the Hamiltonian

$$
H_{1}(\theta, I, \lambda)=\frac{\partial F}{\partial \lambda}(q(\theta, I, \lambda), I, \lambda) .
$$

As a consequence, in the action-angle variables the modulated map (1) can be written in the form (3), hence proving the statement made in Sec. II. Such a map can be represented as a shift along trajectories of a two-frequency system [40], and the theory for two-frequency systems is applicable for this map. To prove the adiabatic invariance of the action $I$ we apply a perturbative approach that introduces new action-angle variables $(\phi, J)$

$$
\begin{aligned}
& \theta=\phi+\epsilon \frac{\partial G}{\partial J}(\phi, J, \lambda)+O\left(\epsilon^{2}\right), \\
& I=J+\epsilon \frac{\partial G}{\partial \phi}(\phi, J, \lambda)+O\left(\epsilon^{2}\right)
\end{aligned}
$$

to reduce map (3) to an integrable form up to terms of order $O\left(\epsilon^{2}\right)$. By changing variables we obtain a homological equation to define $G(\phi, J)$

$$
G(\phi, J, \lambda)-G(\phi-\Omega(J, \lambda), J, \lambda)=H_{1}(\phi, J, \lambda),
$$

where $\Omega(J, \lambda)=\partial H(J, \lambda) / \partial J$. According to [3], the following equality holds:

$$
\left.\frac{\partial F}{\partial \lambda}\right|_{q, I}(\theta, I)=-\frac{1}{\Omega(E, \lambda)} \int^{\theta}\left(\left.\frac{\partial H}{\partial \lambda}\right|_{q, p}-\left\langle\left.\frac{\partial H}{\partial \lambda}\right|_{q, p}\right\rangle\right) d \theta,
$$

where \langle\rangle is the average value with respect to the angle variable. Then one can prove

$$
\left\langle\left.\frac{\partial F}{\partial \lambda}\right|_{q, I}(\theta, I)\right\rangle=0
$$

and using the Fourier expansion

$$
\left.\frac{\partial H}{\partial \lambda}\right|_{q, p}-\left\langle\left.\frac{\partial H}{\partial \lambda}\right|_{q, p}\right\rangle=\sum_{k \neq 0} h_{k}(I, \lambda) e^{i k \theta},
$$

one computes

$$
\left.\frac{\partial F}{\partial \lambda}\right|_{q, I}(\theta, I)=-\sum_{k \neq 0} h_{k}(I, \lambda) \frac{e^{i k \theta}}{i k \Omega(I, \lambda)} .
$$

Then, if $\Omega(J, \lambda) \neq 0$, we get a formal solution of Eq. (A11) as

$$
G(\phi, J, \lambda)=\sum_{k \neq 0} \frac{h_{k}(J, \lambda) e^{i k \phi}}{i k \Omega(J, \lambda)\{1-\exp [-i k \Omega(J, \lambda)]\}} .
$$

Note that if we introduce the operator

$$
\mathbb{T}_{\Omega} \sum_{k \neq 0} h_{k}(I, \lambda) e^{i k \theta}=\sum_{k \neq 0} \frac{i k \Omega(I, \lambda)}{1-\exp [-i k \Omega(I, \lambda)]} h_{k}(J, \lambda) e^{i k \theta}
$$

and the new Hamiltonian

$$
\hat{H}_{1}(\theta, I, \lambda)=\mathbb{T}_{\Omega} \frac{\partial F}{\partial \lambda}(\theta, I, \lambda),
$$

the function $G(\phi, J, \lambda)$ satisfies the homological equation

$$
\Omega(J, \lambda) \frac{\partial G}{\partial \phi}(\phi, J, \lambda)=-\hat{H}_{1}(\phi, J, \lambda),
$$

corresponding to the perturbation theory for Hamiltonian systems. This remark is useful to extend the adiabatic theory to slowly modulated area-preserving maps. The operator $\mathbb{T}_{\Omega}$ changes the $\theta$ Fourier components, and its kernel is the average value. Moreover, the following limit holds for any finite and fixed $k \in \mathbb{Z}$ :

$$
\lim _{\Omega \rightarrow 0} \frac{i k \Omega(I, \lambda)}{1-\exp [-i k \Omega(I, \lambda)]}=1,
$$

meaning that, since the frequency $\Omega(I, \lambda)$ vanishes at the separatrix curve, the operator $\mathbb{T}_{\Omega}$ can be extended up to the separatrix curve if finite Fourier series are considered. The operator $\mathbb{T}_{\Omega}$ is defined in an open set of the action variable if there are no resonance conditions (5) for each Fourier component $k$ in the expansion (A15). Therefore, a cutoff $k_{\max }$ has to be introduced in the Fourier expansion (A15), and it should be proved that the remainder is of order $O(\epsilon)$. To this aim it is customary to extend the domain of the definition of $\theta$ to the complex plane in order to make use of the estimates available for analytic functions in $\mathbb{C}$. Assuming that $\partial H / \partial \lambda$ is a bounded analytic function in a strip $|\operatorname{Im} \theta| \leqslant \gamma(I, \lambda)$ such as for a given action value $I$,

$$
\left|\frac{\partial H}{\partial \lambda}\right|_{q, p} \mid \leqslant M,
$$


with $M$ being a constant independent of $I$, then following estimate holds [41]:

$$
\begin{aligned}
& \sum_{|k| \geqslant k_{\max }} \frac{\left|h_{k}(I, \lambda)\right|}{k \Omega(I, \lambda)} \\
& \leqslant e^{-\gamma(I, \lambda) k_{\max }} \sqrt{\sum_{|k| \geqslant k_{\max }}\left|h_{k}(I, \lambda)\right|^{2} e^{2 \gamma(I, \lambda)|k|}} \\
& \times \sqrt{\sum_{|k| \geqslant k_{\max }} \frac{1}{[k \Omega(I, \lambda)]^{2}} \leqslant c M \frac{e^{-\gamma(I, \lambda) k_{\max }}}{k_{\max } \Omega(I, \lambda)}},
\end{aligned}
$$

taking into account the cutoff $k_{\max }$ in the Fourier expansion. Then, by solving the homological equation (A11) neglecting the Fourier components $|k| \geqslant k_{\max }$, the remainder is of order $O(\epsilon)$, provided $k_{\max }$ fulfils the condition

$$
\frac{c M e^{-\gamma(I, \lambda) k_{\max }}}{k_{\max } \Omega(I, \lambda)} \leqslant \epsilon .
$$

We remark that $k_{\max }$ is a function of $I$ and $\lambda$, so that it is defined locally in phase space.

The additional step consists of analyzing the domain of validity of the cutoff introduced earlier. Under generic assumptions the relation $\phi=\Omega t$ suggests the estimate $\gamma(I, \lambda) \simeq$ $\gamma_{0}|\Omega(I, \lambda)|$. Furthermore, one expects that $\gamma_{0}^{-1}$ can be related to $\max |\Omega(J, \lambda)|$ in the considered phase space region, so that from inequality (A19), we obtain the condition

$$
k_{\max }|\Omega(I, \lambda)| \geqslant \chi\left(\gamma_{0}, M, \epsilon\right), \quad \epsilon=\frac{c M e^{-\gamma_{0} \chi}}{\chi} .
$$

The small denominators $1-\exp [-k \Omega(J, \lambda)]$ in the expansion (A15) can be controlled if

$$
k_{\max }|\Omega(J, \lambda)| \leqslant c^{\prime}<2 \pi .
$$

Comparing the constraints on $k_{\max }|\Omega(J, \lambda)|$, we obtain a final condition on $\gamma_{0}$ and $M$ in the form

$$
\chi\left(\gamma_{0}, M, \epsilon\right) \leqslant c^{\prime},
$$

[1] A. Neishtadt, Sov. Phys. Dokl. 20, 189 (1975).

[2] A. Neishtadt, J. Appl. Math. Mech. 39, 594 (1976).

[3] A. Neishtadt, Sov. J. Plasma Phys. 12, 568 (1986).

[4] J. L. Tennyson, J. R. Cary, and D. F. Escande, Phys. Rev. Lett. 56, 2117 (1986).

[5] J. R. Cary, D. F. Escande, and J. L. Tennyson, Phys. Rev. A 34, 4256 (1986).

[6] J. H. Hannay, J. Phys. A 19, L1067 (1986).

[7] A. N. Vasil'ev and M. A. Guzev, Theor. Math. Phys. 68, 907 (1986).

[8] B. V. Chirikov, Proc. R. Soc. London, Ser. A 413, 145 (1987).

[9] Y. Elskens and D. F. Escande, Nonlinearity 4, 615 (1991).

[10] J. Henrard, Dyn. Reported 2, 117 (1993).

[11] Y. Elskens and D. F. Escande, Phys. D (Amsterdam, Neth.) 62, 66 (1993).

[12] B. V. Chirikov and V. V. Vecheslavov, J. Exp. Theor. Phys. 90, 562 (2000) which is satisfied if

$$
\epsilon \geqslant \frac{c M e^{-2 \pi \gamma_{0}}}{2 \pi}
$$

This implies that the adiabatic invariance of the action for such maps cannot hold for arbitrarily small values of $\epsilon$ due to the presence of nonlinear resonances. Nevertheless, once condition (A21) is satisfied, it can be applied in the neighborhood of the separatrix curve since it holds in the limit $\Omega \rightarrow 0, k_{\max } \rightarrow \infty$ with $k_{\max } \Omega \simeq$ const. Therefore, the function

$$
J(\theta, I, \lambda)=I-\epsilon \sum_{k \neq 0}^{|k| \leqslant k_{\max }(\Omega, \epsilon)} \frac{i k h_{k}(J, \lambda) e^{i k \theta}}{1-\exp [-i k \Omega(J, \lambda)]}+O\left(\epsilon^{2}\right)
$$

can be extended up to the separatrix curve in each phase space region. When approaching the separatrix $(\Omega \rightarrow 0)$ the function $J(\theta, I, \lambda)$ tends to the IAI [42] of the interpolating Hamiltonian $H(q, p, \lambda)$ that was introduced in Eq. (2). Hence, once condition (A21) is satisfied, it is possible not only to cast map (3) in the form (4) but also to find that the action $I$ is an adiabatic invariant for the initial dynamics (1) if we are not too close to the separatrix. By performing another perturbative step we can also prove that the new action $J$ is an IAI if we restrict condition (A21) to cutoff terms of order $O\left(\epsilon^{2}\right)$, i.e.,

$$
\epsilon \geqslant \sqrt{\frac{c M}{2 \pi}} e^{-\pi \gamma_{0}} .
$$

Therefore, the application of the adiabatic invariance theory to analytic maps in the neighborhood of an elliptic fixed point is justified for $\epsilon$ values that satisfy condition (A23).

The last key observation is that for the special case of Hamiltonian systems of the form (6), it is reasonable to assume that the parameter $\gamma_{0}$ of Eq. (A21) is expected to be of order $1 / \mu$, where $\mu$ bounds the variation of the monotonic function $\omega_{1}(\lambda)$ introduced in Eq. (8), so that if $\mu$ is small enough, we can apply the adiabatic theory to the time-dependent map, as $\epsilon$ can be chosen to be small.
[13] A. Neishtadt, A. A. Vasiliev, and A. Itin, Phys. D (Amsterdam, Neth.) 141, 281 (2000).

[14] R. Cappi and M. Giovannozzi, Phys. Rev. Lett. 88, 104801 (2002).

[15] R. Cappi and M. Giovannozzi, Phys. Rev. Spec. Top. Accel. Beams 7, 024001 (2004).

[16] S. Gilardoni, M. Giovannozzi, M. Martini, E. Métral, P. Scaramuzzi, R. Steerenberg, and A.-S. Müller, Phys. Rev. Spec. Top. Accel. Beams 9, 104001 (2006).

[17] A. Franchi, S. Gilardoni, and M. Giovannozzi, Phys. Rev. Spec. Top. Accel. Beams 12, 014001 (2009).

[18] A. Neishtadt, Sov. Phys. Dokl. 32, 571 (1987).

[19] A. Neishtadt, Celestial Mech. Dyn. Astron. 65, 1 (1997).

[20] S. Sridhart and J. Touma, Mon. Not. R. Astron. Soc. 279, 1263 (1996).

[21] Q. Niu, Phys. Rev. B 34, 5093 (1986). 
[22] O. Entin-Wohlman, A. Aharony, and Y. Levinson, Phys. Rev. B 65, 195411 (2002)

[23] J. P. Pekola, J. J. Toppari, M. Aunola, M. T. Savolainen, and D. V. Averin, Phys. Rev. B 60, R9931 (1999).

[24] V. I. Arnol'd, Dokl. Akad. Nauk SSSR 161, 9 (1965).

[25] V. Arnol'd, V. Kozlov, and A. Neishtadt, Mathematical Aspects of Classical and Celestial Mechanics (Springer, Heidelberg, 2006).

[26] M. Hénon, Q. Appl. Math. 27, 291 (1969).

[27] A. Bazzani G. Servizi, E. Todesco, and G. Turchetti, CERN, Yellow Report No. 94-02, 1994 (unpublished).

[28] A. Bazzani, S. Marmi, and G. Turchetti, Celestial Mech. Dyn. Astron. 47, 333 (1990).

[29] G. D. Birkhoff, Acta Math. 43, 1 (1920).

[30] A. Bazzani, M. Giovannozzi, G. Servizi, E. Todesco, and G. Turchetti, Phys. D (Amsterdam, Neth.) 64, 66 (1993).

[31] A. Bazzani, F. Brini, and G. Turchetti, in Workshop on Nonlinear and Collective Phenomena in Beam Physics, AIP Conf. Proc. No. 395 (AIP, New York, 1997), p. 129.
[32] A. Bazzani and F. Brini, in Hamiltonian Systems with Three or More Degrees of Freedom, edited by C. Simó, NATO ASI Series Vol. 533 (Springer, Dordrecht, 1999), p. 300.

[33] D. L. Bruhwiler and J. R. Cary, Phys. D (Amsterdam, Neth.) 40, 265 (1989).

[34] M. Aiba, S. Machida, Y. Mori, and S. Ohnuma, Phys. Rev. Spec. Top. Accel. Beams 9, 084001 (2006).

[35] M. Giovannozzi and J. Morel, Phys. Rev. Spec. Top. Accel. Beams 10, 034001 (2007).

[36] D. Vainchtein and I. Mezić, Phys. Rev. Lett. 93, 084301 (2004).

[37] A. Neishtadt, Chaos 1, 42 (1991).

[38] A. I. Neishtadt, Selecta Math. Sov. 12, 195 (1993).

[39] M. Giovannozzi, D. Quatraro, and G. Turchetti, Phys. Rev. Spec. Top. Accel. Beams 12, 024003 (2009).

[40] A. Neishtadt, Proc. Steklov Inst. Math. 250, 183 (2005).

[41] H. Rüssmann, Celestial Mech. Dyn. Astron. 14, 33 (1976).

[42] V. I. Arnol'd, Dokl. Akad. Nauk SSSR 142, 758 (1962). 